\title{
SOLID STATE MODULATOR APPLICATIONS IN LINEAR ACCELERATORS
}

\author{
M.P.J. Gaudreau, J.A. Casey, T.P. Hawkey, J.M. Mulvaney, M.A. Kempkes, P. Ver Planck \\ Diversified Technologies, Inc. 35 Wiggins Ave., Bedford, MA 01730
}

\section{ABSTRACT}

The next generation of linear colliders will require an order of magnitude leap in pulsed power to millions of volts at thousands of amperes, delivered at much higher efficiency than is presently available. The current technology base of thyratrons, PFNs, etc., is inherently limited in scaling to meet these new requirements.

Diversified Technologies, Inc. (DTI), has had tremendous success since 1993 in the application of high

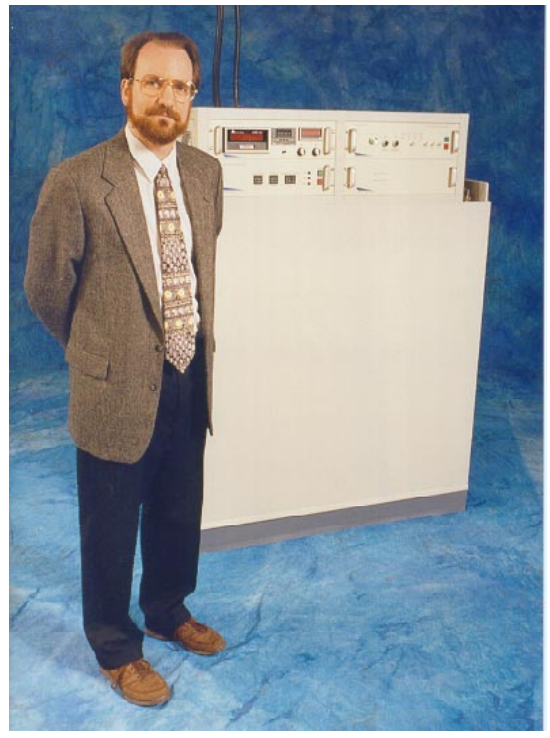

Figure 1 DTI's HVPM 100-500 100 kV, 50 MW Peak Pulse Modulator.

voltage IGBT devices to large, high-voltage and highcurrent modulator systems. DTI has sold commercial solid-state modulators capable of 20 to $160 \mathrm{kV}$ and 150 to 2000 A for customer applications ranging from RF tube testing to ion-implantation. This technology is rapidly becoming the preferred alternative to conventional vacuum tube modulators and switches for future accelerator designs.

\section{BACKGROUND}

\subsection{IGBTs}

Solid state devices are, in general, low voltage devices. Recent advances in Insulated Gate Bipolar Transistors (IGBTs) have improved the voltage and current handling characteristics considerably. Typical devices have voltage ratings from $1200 \mathrm{~V}-3300 \mathrm{~V}$ and current ratings from 50A-1200A continuous. They also feature the very low drive current requirements of Field Effect Transistors (thus the Insulated Gate). This eliminates the need for cascaded stages of bipolar drives required by the low betas of early high current bipolar circuit designs.

To use IGBTs for high voltage switching, many devices

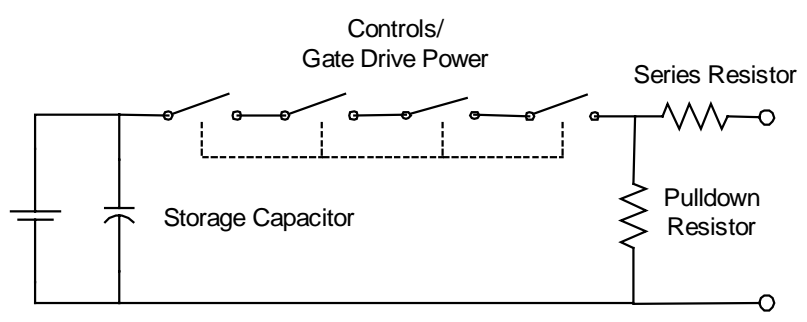

Figure 2: Solid State Modulator Components

must be cascaded in series. (Figure 2). This concept provides the flexibility of a modular design, with no inherent limit to voltage handling. However, it also necessitates the formidable task of ensuring that the load is shared equally between devices so that no single device sees harmful or destructive voltages. The gate drives must be highly synchronized to accomplish this. DTI has developed and patented the technology to achieve this synchronization, which has been demonstrated at up to 160 IGBTs in series, and up to six IGBTs in parallel.

The benefits of solid-state switching of high voltage include high reliability, significantly higher efficiency, and fast, repeatable switching characteristics. Our analyses have shown that these solid state systems are capable of providing $15 \mathrm{x}$ improvements in power efficiency during switching, and orders of magnitude improvements in reliability compared to vacuum tube and thyratron based switches.

\section{SOLID STATE MODULATOR PRINCIPLES}

\subsection{Simple Switch-Ideal Pulse}

Ideally, a modulator acts as a simple switch between a high voltage power supply and its load (such as a klystron). The desired properties of such an ideal switch would be infinite voltage holdoff, infinite off-resistance, zero on-resistance, and full immunity to transients and voltage reversals. 
Achieving, as closely as possible, this ideal pulse is critical to the performance of a number of pulsed power applications. Generating pulses which closely approach this ideal pulse is typically a critical objective of high pulsed power system design.

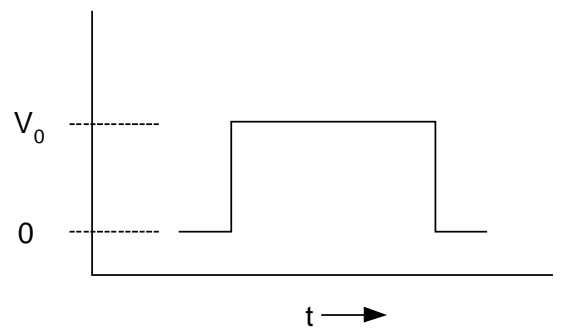

Figure 3: Ideal Pulse

Historically, vacuum switch tubes or thyratrons, alone, or in combination with Pulse Forming Networks (PFNs) and pulse transformers, have been used for this purpose. The non-ideal behavior of these conventional

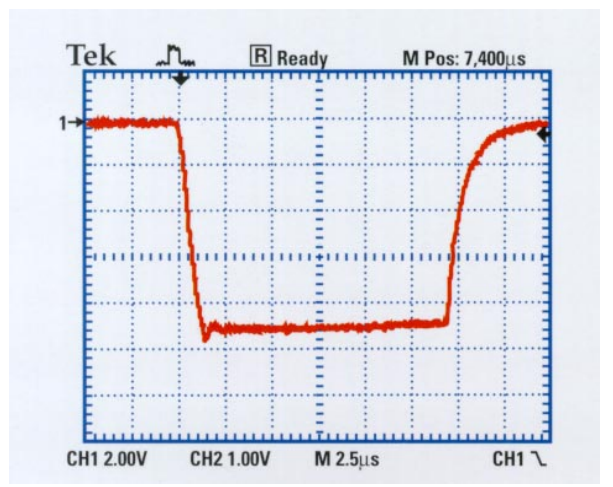

Figure 4: Nearly Ideal HVPM 100-500

Pulse 80 kV, 90A Into Water Resistor

switches includes a large effective voltage drop, limited current capability and speed, high maintenance, and complex driving and protection circuitry. As future system requirements extend to higher voltage and power, the use of vacuum tubes becomes increasingly impractical due to the inherent voltage and current limits of these devices.

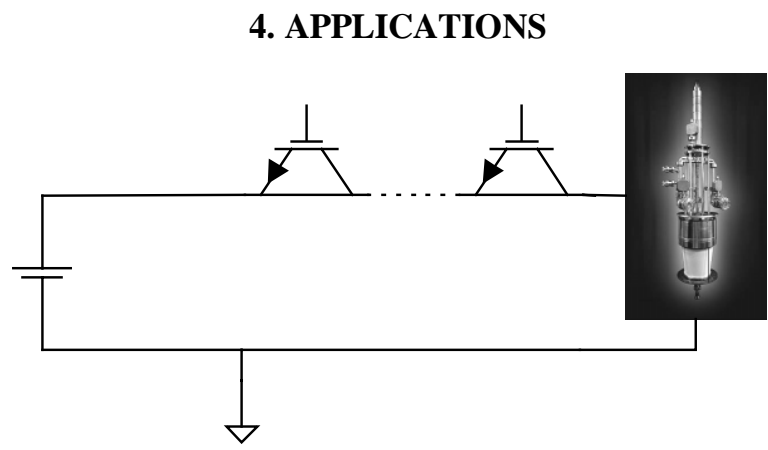

Figure 5: Cathode Modulator / Crowbar Replacement

\subsection{Cathode Modulator}

There are two principal approaches to applying high voltage solid-state modulators to accelerators. First, very high voltage and moderate current operation can be used to switch microwave devices directly. This also has the direct benefit eliminating the need for a separate crowbar circuit, since the switch can open rapidly in the event of an arc. Alternately, lower switch voltages at high currents can be used in conjunction with pulse transformers to provide pulses of energy. In either case, very fast risetimes and falltimes are required for operational and cost efficiency reasons. Furthermore, these basic circuits can be used either as cathode modulators or mod-anode modulators in typical accelerator systems.

\subsection{Crowbar Replacement}

The response time of DTI's technology provides a new level of protection to sensitive high power electronics, such as vacuum tubes. A typical crowbar, at $100 \mathrm{kV}$ and 100 A current, will allow $20 \mathrm{~J}$ of energy through the arc even with a fast $2 \mu \mathrm{S}$ response- below the $50 \mathrm{~J}$ which may cause damage, but enough to degrade the lifetime of the tube.

DTI's switches can open and close in less than $0.5 \mu \mathrm{S}$. This fast response allows these switches to operate as effective 'fast fuses'. Under the conditions described above, the energy available to cause tube damage is reduced by $75 \%$ to $5 \mathrm{~J}$. Unlike a typical crowbar, however, the solid state switch can be closed again almost immediately. Furthermore, this cycle can be repeated indefinitely.

\subsection{Mod-Anode Modulator}

The same switching solid state switching technology can be applied to mod-anode modulators - either directly (hard switched) or in conjunction with a pulse

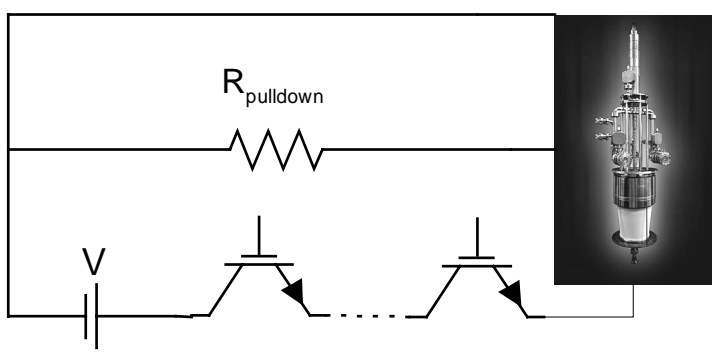

Figure 6: Mod-Anode Modulator

transformer. Typically, the lower power required for modanode modulation allows very fast operation. DTI has demonstrated a $20 \mathrm{kV}$ mod-anode modulator for a Navy radar application capable of $400 \mathrm{kHz}$ operation. 


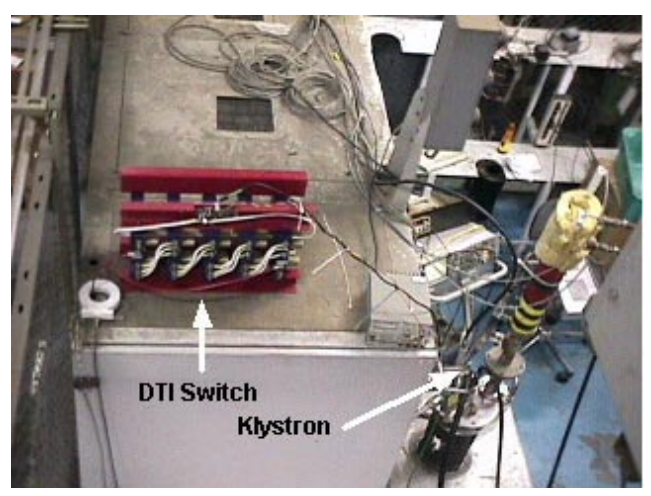

Figure $7: 45$ kV, 30 a DTI Solid State Switch with Klystron at SLAC

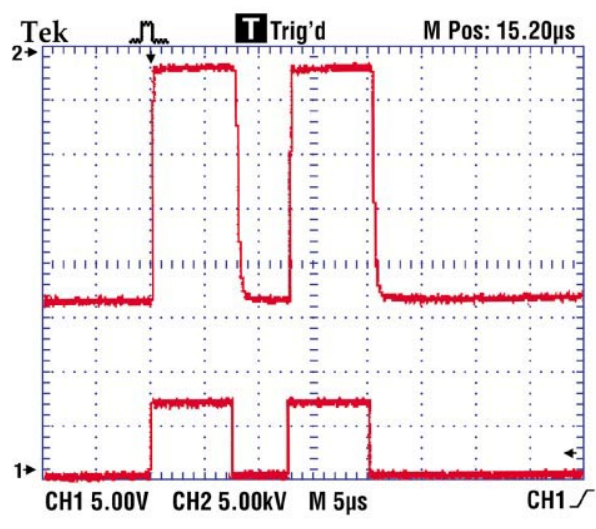

Figure 8: SLAC Prototype $45 \mathrm{kV}, 30 \mathrm{~A}$ Switch Result @ 22 kV, 80 a Into Resistive Load

\section{BENEFITS}

The primary benefit of a full voltage (cathode) switch for advanced accelerators will be economic. The principal power loss in a direct switched system is the $f C V^{2}$ losses to the parasitic capacitance. The fast risetime capability of a direct switch will reduce power lost on ramp-up and settling time. A PFN or hybrid modulator/transformer approach will generally have a slower risetime because of series inductance, as well as additional magnetizing current and parasitic capacitance in the magnetics. This also allows fully programmable pulse-width, and the adaptability of the accelerator system to varying physics requirements.

\subsection{Increased Efficiency}

Switch tubes have an inherently high voltage drop across them. At high peak power, this results in significant power loss across the tube, and drives the need for substantial cooling systems for the tube itself.

\subsection{Higher Reliability}

Due to arcing and emissions/outgassing within the tube itself and short cathode life, vacuum tubes have an inherently limited lifetime. Although improvements have been made in tube manufacturing since WWII, tube lifetime is still limited to the thousands (tens of thousands in some case) of hours.

DTI's solid state modulators provide nearly infinite flexibility in pulse parameters (pulsewidth, PRF, peak current, voltage, and duty cycle). Typical rise / fall times for DTI's PowerMod ${ }^{\mathrm{TM}}$ Systems are $<0.5 \mu \mathrm{S}$ for high power (MWs) pulses.

Line modulators and PFNs use resonant circuits to achieve high power pulses. These systems are designed to provide a very narrow range of PRFs and pulsewidths.

\subsection{Increased Current Handling Capability}

With DTI's solid state modulators, maximum current capability is a design parameter. DTI has built modulators capable of handling 2000A, and placing switch modules in parallel can accommodate higher currents.

\subsection{Ruggedness}

DTI's PowerMod ${ }^{\mathrm{TM}}$ solid state modulators are inherently rugged, isolated from external conditions, and able to provide consistent performance over a wide range of conditions. High voltage tubes, by virtue of their construction, are relatively fragile and susceptible to variations in performance due to operating conditions.

\subsection{Safety from $X$-rays}

High voltage power tubes produce $\mathrm{X}$-rays due to inherent grid emission leakage or primary electron conduction. Shielding of the tube is required for safe operation. DTI's PowerMod $^{\mathrm{TM}}$ systems do not create X-rays.

The combination of the technology required for advanced accelerators, and DTI's ongoing efforts to move to higher voltages, peak power, and frequency, provides both our government and commercial customers significant advantages in future high voltage, high power systems.

(wd) 